\title{
Single agent daratumumab in advanced multiple myeloma possesses significant efficacy even in an unselected "real-world" population
}

\author{
Jiri Minarik ${ }^{\mathrm{a}}$, Ludek Pour ${ }^{\mathrm{b}}$, Vladimir Maisnarc, Ivan Spicka ${ }^{\mathrm{d}}$, Alexandra Jungova ${ }^{\mathrm{e}}$, Tomas Jelinek', Lucie Brozova ${ }^{\mathrm{g}}$, \\ Petra Krhovska ${ }^{a}$, Vlastimil Scudla ${ }^{a}$, Roman Hajek ${ }^{f}$
}

\begin{abstract}
Objective. The treatment of relapsed and refractory multiple myeloma (RRMM) remains challenging. The outcomes in highly pretreated populations are unsatisfactory and there is urgent need for novel and safe therapeutic approaches. Recently, daratumumab has been approved for RRMM with promising results even in monotherapy. The aim of this study was to assess the efficacy of single agent daratumumab outside a clinical trial.

Patients and Methods. 14 patients with RRMM and significant pretreatment (median 4.5 previous lines) entered a specific healthcare program and received treatment with single agent daratumumab. They were followed for therapeutic response based on IMWG criteria, and incidence of adverse events. The data were collected using the Registry of Monoclonal Gammopathies.

Results. The overall response rate was $38.5 \%$. $23.1 \%$ of patients reached very good partial response, $15.4 \%$ reached partial remission, $15.4 \%$ had minimal response, $38.5 \%$ had stable disease and $7.7 \%$ had progressive disease. The median progression free survival was 4.6 months and median overall survival was not achieved. The toxicities were mostly mild, only infectious complications and hematological toxicity reached grade III.

Conclusion. We conclude that daratumumab has significant activity in highly pretreated RRMM even as a single agent, with an acceptable toxicity profile and survival impact.
\end{abstract}

Key words: multiple myeloma, relapsed and refractory, daratumumab, progression free survival, overall survival

Received: May 15, 2018; Revised: July 25, 2018; Accepted: October 12, 2018; Available online: November 6, 2018

https://doi.org/10.5507/bp.2018.064

(c) 2019 The Authors. This is an open access article licensed under the Creative Commons Attribution License

(https://creativecommons.org/licenses/by/4.0/).

${ }^{a}$ Department of Hemato-oncology, University Hospital Olomouc and Faculty of Medicine, Palacky University Olomouc, Czech Republic ${ }^{b}$ Department of Internal Medicine, Hematology and Oncology, University Hospital Brno, Czech Republic

'Department of Clinical Hematology, University Hospital Hradec Kralove, Czech Republic

${ }^{d}$ Department of Internal Medicine, University Hospital Praha, Czech Republic

${ }^{e}$ Department of Haemato-Oncology, University Hospital Pilzen, Czech Republic

${ }^{f}$ Department of Haematooncology, University Hospital Ostrava and Faculty of Medicine, University of Ostrava, Czech Republic;

IInstitute of Biostatistics and Analyses, Faculty of Medicine, Masaryk University Brno, Czech Republic

Corresponding author: Jiri Minarik, e-mail: abretina@email.cz

\section{INTRODUCTION}

Daratumumab (DARA) is an anti-CD38 monoclonal antibody $(\mathrm{mAb})$ recently introduced in the treatment of relapsed or relapsed and refractory multiple myeloma (MM). DARA targets myeloma cells via a variety of immune-mediated mechanisms including complementdependent cytotoxicity, antibody-dependent cell-mediated cytotoxicity, antibody-dependent cellular phagocytosis and direct apoptosis with crosslinking ${ }^{1,2}$. DARA was originally chosen from a large panel of anti-CD38 mAbs because of its ability to trigger complement-dependent cytotoxicity $^{3}$. Another mechanism of action - immunomodulatory - has recently been discovered. This though still not fully understood, is probably based on depletion of CD38+ immune suppressive cells such as Tregs, Bregs or myeloid derived suppressor cells ${ }^{1}$.

DARA was approved by the US Food and Drug Administration (FDA) as a single agent for advanced $\mathrm{MM}$, based on the results of the phase II trial SIRIUS ${ }^{4}$.
In the SIRIUS trial, DARA was used as a single agent treatment for advanced multiple myeloma. Even in this setting of highly pre-treated MM (median previous treatment lines was 5) it had considerable treatment response with prolongation of progression free survival (PFS) and overall survival (OS).

Based on the SIRIUS trial, a state based healthcare program (54767414MMY4001) was introduced into the Czech Republic in March 2016 to assess the efficacy of DARA in a "real world" setting. We present the results of the program.

\section{PATIENTS AND METHODS}

The healthcare program was registered at the Ministry of Health of the Czech Republic under the identification code 54767414MMY4001. The program was conducted under the aegis of the Czech State Institute for Drug Control (SUKL - Státní Ústav pro Kontrolu Léčiv) and carried 
out at 7 hematological centres in the Czech Republic. Altogether, a maximum of 14 patients were intended for recruitment and a maximum of 2 patients at each centre. The inclusion criteria followed FDA approval criteria, ie. "DARZALEX is a human CD38-directed monoclonal antibody indicated for the treatment of patients with multiple myeloma who have received at least three prior lines of therapy including a proteasome inhibitor (PI) and an immunomodulatory agent or who are double-refractory to a PI and an immunomodulatory agent."(ref.5).

The patients received daratumumab monotherapy according to the schedule of SIRIUS trial: DARA $16 \mathrm{mg} /$ $\mathrm{kg}$ i.v. per week for 8 weeks (cycles 1 and 2), then every 2 weeks for 16 weeks (up to cycle 6 ), and every 4 weeks thereafter. First infusions were $1000 \mathrm{~mL}$ at $50 \mathrm{~mL} / \mathrm{h}$, followed by dose escalation up to $200 \mathrm{~mL} / \mathrm{h}$. Second and subsequent infusions were $500 \mathrm{~mL}$ at $100 \mathrm{~mL} / \mathrm{h}$ with an increase to $200 \mathrm{~mL} / \mathrm{h}$. Standard premedication was used before DARA administration: methylprednisolone 100 $\mathrm{mg}$ intravenously for the first and second infusions and $60 \mathrm{mg}$ thereafter, or equivalent (mostly we used dexamethasone 16-40 mg), paracetamol 650-1000 mg and an antihistamine drug according to local guidelines. The data were collected using the Registry of Monoclonal Gammopathies (RMG), a large multicenter database for collecting the data of MM patients within Central Europe ${ }^{6}$.

\section{RESULTS}

Despite a very short recruitment period (practically within one month), all 14 patients were recruited and started DARA treatment. The basic demographics, group and disease characteristics were standard and are summa-

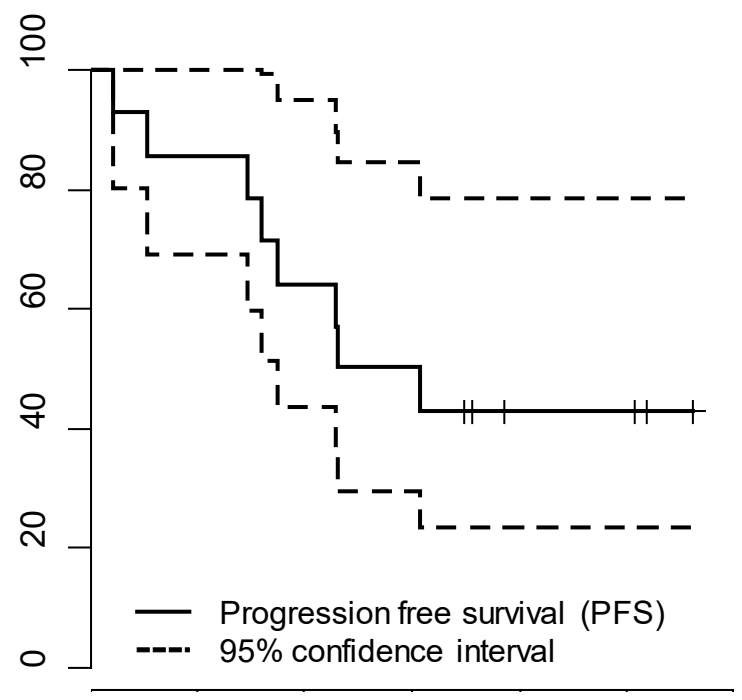

PFS

median $(95 \% \mathrm{CI})$

3 months $(95 \% \mathrm{CI})$

6 months $(95 \% \mathrm{CI})$ rized in Table 1. Median age was 60.5 years with an even distribution of males and females. Most of the patients were ECOG 1 (8/14 patients, 57.1\%), followed by ECOG 0,2 and 3 (in $2 / 14$ patients each, $14.3 \%$ ). ISS stage at the time of relapse before DARA treatment was assessed in 12 patients, most of them being stage $3(42.9 \%)$, followed by ISS 1 and 2 (both 21.4\%). The initial ISS (at the time of MM diagnosis) was assessed in all patients, and was as follows: ISS 1 in $7 / 14$ patients (50\%), ISS 2 in $4 / 14$ patients $(28.6 \%)$ and ISS 3 in $3 / 14$ patients $(21.4 \%)$. The patients had mildly elevated levels of creatinine (median $113 \mathrm{umol} / \mathrm{L}$, up to $250 \mathrm{umol} / \mathrm{L}$ ) and $21.4 \%$ of patients (3/14) had extramedullary disease, with a significant percentage of patients having high-risk cytogenetic features, $\mathrm{t}(4 ; 14)$ in $5 / 7$, del17 in $1 / 6$, del13 in $7 / 8$ and amp1q21 in $3 / 6$ patients. The median previous lines was $4.5(2-8), 93 \%$ of patients had previous autologous stem cell transplant (ASCT), all received previous bortezomib, most of them received lenalidomide ( $86 \%)$ and thalidomide $(64 \%)$, and a significant percentage received pomalidomide $(50 \%)$ and carfilzomib (21\%). Two thirds of the patients were refractory to their last line of treatment before DARA. Median follow up for the whole cohort was 8 months.

The treatment responses and intervals are shown in Table 2 together with indirect comparison with the SIRIUS trial. The overall response rate (ORR) was $38.5 \%$ with $23.1 \%$ of patients reaching very good partial response (VGPR) or better, 15.4\% reached partial remission (PR), $15.4 \%$ of SD patients had minimal response (MR), $38.5 \%$ had stable disease (SD), and 7.7\% had progressive disease (PG). The overall PFS was 4.6 months and median OS was not reached (Fig 1). The toxicities within DARA treatment were as follows: hematological toxities - anemia (grade I in $7.1 \%$, gr II in $35.7 \%$, gr III in $7.1 \%$ ), thrombocytopenia (gr I in $7.1 \%$, gr II in $7.1 \%$, gr III in $28.6 \%$ ), neutro-

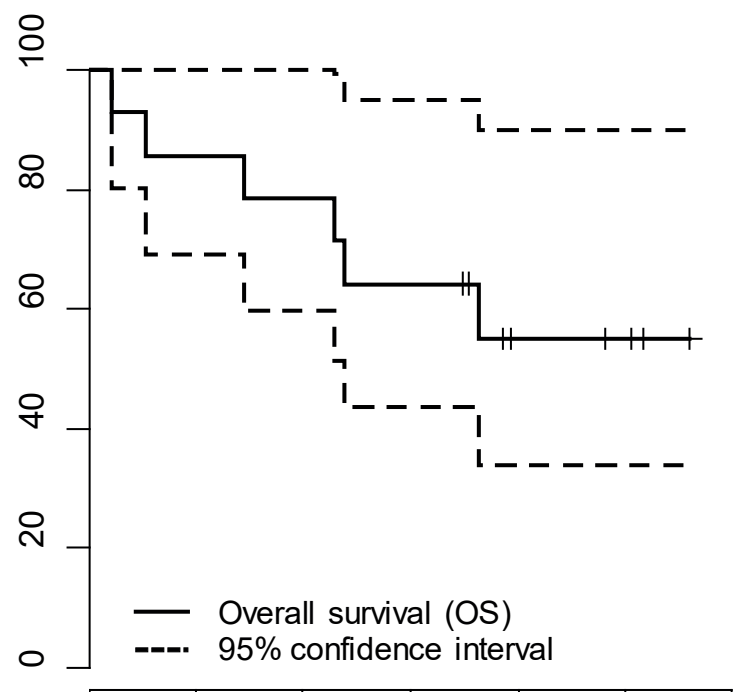

$\begin{array}{lc}\text { OS from start of DARA } & \mathrm{n}=14 \\ \text { median }(95 \% \mathrm{CI}) & \text { not reached } \\ 3 \text { months }(95 \% \mathrm{CI}) & 78.6(47.3-92.5) \\ 6 \text { months }(95 \% \mathrm{CI}) & 64.3(34.3-83.3)\end{array}$

Fig. 1. Progression free survival and overall survival in advanced relapsed and/or refractory multiple myeloma treated by daratumumab monotherapy. 
Table 1. Basic demographics and group characteristics.

\begin{tabular}{|c|c|}
\hline Male : Female ratio & $1: 1$ \\
\hline Age - median (range) & 60.5 years $(37.0-80.0)$ \\
\hline Patients over 70 years & $21.4 \%$ \\
\hline \multirow[t]{3}{*}{ ISS stage at the time of multiple myeloma diagnosis } & Stage $150.0 \%(7 / 14)$ \\
\hline & Stage $228.6 \%(4 / 14)$ \\
\hline & Stage $321.4 \%(3 / 14)$ \\
\hline \multirow[t]{4}{*}{ ISS stage at relapse before daratumumab treatment } & Stage $121.4 \%(3 / 14)$ \\
\hline & Stage $221.4 \%(3 / 14)$ \\
\hline & Stage $342.9 \%(6 / 14)$ \\
\hline & Unknown $14.3 \%(2 / 14)$ \\
\hline \multirow[t]{4}{*}{ Performance status } & ECOG 0 14.3\% (2/14) \\
\hline & ECOG $157.1 \%(8 / 14)$ \\
\hline & ECOG $214.3 \%(2 / 14)$ \\
\hline & ECOG 3 14.3\% (2/14) \\
\hline Extramedullary disease & $21.4 \%(3 / 14)$ \\
\hline Creatinin clearance - median (range) & 113 umol/L (63-260 umol/L) \\
\hline \multirow[t]{4}{*}{ Cytogenetics } & $t(4 ; 14)=5$ out of 7 \\
\hline & del17= 1 out of 6 \\
\hline & del13= 7 out of 8 \\
\hline & amp1q21 $=3$ out of 6 \\
\hline \multicolumn{2}{|l|}{ Previous treatment } \\
\hline Previous treatment lines - median (range) & $4.5(2-8)$ \\
\hline previous ASCT & $93 \%(13 / 14)$ \\
\hline previous bortezomib & $100 \%(14)$ \\
\hline previous carfilzomib & $21 \%(3 / 14)$ \\
\hline previous thalidomid & $64 \%(9 / 14)$ \\
\hline previous lenalidomide & $86 \%(12 / 14)$ \\
\hline previous pomalidomide & $50 \%(7 / 14)$ \\
\hline \multirow[t]{2}{*}{ Refracterity to the last treatment } & Relapsed $33.3 \%$ \\
\hline & Relapsed and refractory $66.7 \%$ \\
\hline
\end{tabular}

ISS - International Staging System; ECOG - Eastern Cooperative Oncology Group performance status; ASCT - autologous stem cell transplant

Table 2. Response rates and intervals in daratumumab monotherapy and indirect comparison with SIRIUS trial ${ }^{3}$.

\begin{tabular}{lcc}
\hline & SIRIUS trial & $\begin{array}{c}\text { Specific } \\
\text { Healthcare Program }\end{array}$ \\
\hline ORR (\%) & 29.2 & 38.5 \\
CBR (\%) & 34 & 53.8 \\
SCR (\%) & 2.8 & 0 \\
CR (\%) & 0 & 0 \\
VGPR (\%) & 9.4 & 23.1 \\
$\geq$ VGPR (\%) & 12.3 & 23.1 \\
PR (\%) & 17 & 15.4 \\
MR (\%) & 4.7 & 15.4 \\
SD (\%) & 43.4 & 38.5 \\
PG (\%) & 17 & 7.7 \\
PFS & 3.7 months & 4.6 months \\
OS & not reached & not reached \\
\hline
\end{tabular}

ORR - overall response rate ( $\geq \mathrm{PR}), \mathrm{CBR}$ - clinical benefit rate $(\geq \mathrm{MR})$, sCR - stringent complete response, CR - complete response, VGPR - very good partial response, PR - partial response, MR - minimal response, $\mathrm{SD}$ - stable disease, $\mathrm{PG}$ - progressive disease, PFS - progression free survival, OS - overall survival penia (gr I in $21.4 \%$, gr III in $21.4 \%$ ). Non-hematological toxicities were predominantly infectious complications (gr II in $35.7 \%$, gr III in $21.4 \%$ ), followed by weakness and fatigue (gr I in $21.4 \%$, gr II in $21.4 \%$, gr III in $7.1 \%$ ), nausea ( $\mathrm{gr}$ I in $14.3 \%$, gr II in $14.3 \%$ ), diarrhea ( $\mathrm{gr}$ I in $21.4 \%$ ), and thrombosis (gr II in $7.1 \%$ ). Infusion related reactions (IRRs) were noticed in $35.7 \%$ (gr I in $7.1 \%$, gr II in $14.3 \%$ and gr III in $14.3 \%$ ).

\section{DISCUSSION}

DARA was approved based on a phase II clinical trial (SIRIUS) as a breakthrough therapy, which is quite rare as most drugs have to confirm their efficacy in phase III trials. Even in the setting of highly pre-treated MM (median previous treatment lines was 5 with most patients being double or triple refractory to proteasome inhibitors and immunomodulators) DARA had substantial treatment response $(\mathrm{ORR}=29.2 \%)$ with prolongation of PFS (3.7 months in the whole cohort, 15 months in DARA responders) and overall survival (median OS not reached, after longer follow-up OS = 20 months) $\left(\right.$ ref. $\left.^{4}\right)$. Other recently approved drugs such as elotuzumab or panobinostat 
do not possess single agent activity. Monotherapy with pomalidomide or carfilzomib reached similar PFS (ranging from 3.7 to 4.6 months) as DARA, but median OS in these studies ranged from 10.2 to 15.4 months which is less than DARA (20.1 months) (ref., ${ }^{7,}$. Moreover, adjusted comparison of real world data from RMG with pooled analysis of daratumumab monotherapy confirmed the same finding ${ }^{9,10}$.

The treatment of advanced stage MM is very exacting. Patients usually experience adverse events from previous treatments, and may have limited bone marrow reserves. Moreover, after several different therapeutic regimens, the underlying MM clone that causes relapse is usually more aggressive and resistant to multiple drug classes. Emerging new treatments are therefore needed to target different pathways and overcome the resistance of the clone. The effectiveness of any single agent is therefore very encouraging in late stage MM but it has to be confirmed by further trials as well as in routine practice. Steroids are among the most effective drugs in MM treatment, especially in combination regimens. Their efficacy as monotherapy in late stage MM is, however, very limited. In a recent review of DARA trials, the drug was evaluated as generally well tolerated as monotherapy and with a manageable tolerability profile when used in combination therapy ${ }^{11}$.

The outcomes of two other phase III clinical trials, CASTOR and POLLUX, that combined daratumumab with bortezomib or lenalidomide and $\mathrm{A}$ in a relapsed setting revealed an unprecedented efficacy of the combined regimens ${ }^{12,13}$. The combination of DARA with other agents definitely has better potential to control the disease than monotherapy but the economic burden of the combinations is not negligible. A single dose of DARA exceeds 7000 USD: Hence, the total cost is highest in the first two months with weekly dosing, decreasing with further treatment with reduced frequency of dosing (once per 14 days cycles $3-6$, once per month thereafter). The cost of combined regimens is increased accordingly. However, recent updates of CASTOR and POLLUX trials after longer follow-up confirm significant benefits with respect to PFS, ORR and depth of response including the rate of minimal residual disease (MRD), with no further safety issues, justifying the increased costs of these regimens ${ }^{14,15}$. Moreover, patients relapsing in the DARA arm responded more favorably to subsequent therapy ${ }^{15}$. It is very likely that DARA plays a similar role in MM treatment as rituximab in B-cell non-Hodgkin lymphomas. Currently, DARA is being tested in first line treatment and even in smoldering MM with very promising results ${ }^{16,17}$. Clinical trials, however, have strict inclusion and exclusion criteria, and a considerable number of patients do not fulfill them. For this reason, the outcomes of clinical trials are usually significantly better than in later common use of the drug. In our program, patients with low performance status and low life-expectancy or significant co-morbidity were included. Despite these inclusions, the results were surprisingly better than in the SIRIUS trial with a significant number of patients achieving therapeutic response, and with indisputable improvement in PFS and OS. The treatment was well tolerated and none of the patients quit the program due to toxicity. However, we need further tools to better define the group of patients who would profit from DARA monotherapy.

We acknowledge the very limited number of patients entering the program. However, the spectrum of patients including demographics, previous treatment and cytogenetics was very similar to that published in the SIRIUS trial though we had a larger number of high-risk patients including those with extramedullary disease. We had fewer less patients with previous carfilzomib pretreatment (21.4\% vs 50\% in the SIRIUS trial). Our results show that the effect of DARA in MM is indisputable. Several issues are still to be resolved including the long-term outcomes when DARA is used in earlier treatment lines, the unexpectedly good synergy of DARA and lenalidomide or possible down-regulation of CD38 and the occurrence of CD38-negative relapses after DARA as reported in our previous paper $^{18}$.

\section{CONCLUSION}

We conclude that single agent daratumumab has significant efficacy in advanced multiple myeloma. The unique mechanism of action via immune-mediated mechanisms provides a novel therapeutic approach with effect in highly pre-treated refractory populations with myeloma clone resistant to other therapies. Even in an unselected real-world population (outside clinical trials) the treatment leads to high response rates as well as to fair prolongation of progression free and overall survival. Our results including response rates and PFS are even better than in the registration trial, confirming the safety and unique efficacy of the regimen. In patients who are refractory to proteasome inhibitors and IMiDs, who would be otherwise left for palliative care, DARA represents a chance for therapeutic response and improvement of both, survival and quality of life. Moreover, patients responding to DARA may recover sensitivity to other drugs, and could benefit from re-treatment or further treatment with other novel agents in the case of following relapse. The economic burden of the regimen is substantial. Nevertheless, our results confirm that the effectiveness of DARA and especially the impact on overall survival even in unselected advanced myeloma patients outweighs the economical impact of this treatment.

Acknowledgement: Supported by the grant AZV 1729343A, IGA-LF-2018-004 and MH CR - RVO (FNOl, 00098892).

Author contributions: JM: manuscript writing, study design and participated in patient recruitment; JM, LP, VM, IS, AJ, TJ, TP, VS, RH: participated in patient recruitment at the 7 hematological centers in the Czech Republic; JM, VM, IS, AJ, TJ, RH: data analysis and critical revision; LB: statistical analysis.

Conflict of interest statement: The authors have no con- 
flict of interest. We thank Janssen-Cilag company for introducing the Patient Named Program into the Czech Republic and for supplies of the daratumumab used.

\section{REFERENCES}

1. Krejcik J, Casneuf T, Nijhof IS, Verbist B, Bald J, Plesner T, Syed K, Liu K, van de Donk NWCJ, Weiss BM, Ahmadi T, Lokhorst HM, Mutis T, Sasser AK. Daratumumab depletes CD38+ immune regulatory cells, promotes T-cell expansion, and skews T-cell repertoire in multiple myeloma. Blood 2016;128:384-94.

2. Jelinek T, Hajek R. Monoclonal antibodies - A new era in the treatment of multiple myeloma. Blood Rev 2016;30:101-10.

3. de Weers M, Tai Y-T, van der Veer MS, Bakker JM, Vink T, Jacobs DC, Oomen LA, Peipp M, Valerius T, Slootstra JW, Mutis T, Bleeker WK, Anderson KC, Lokhorst HM, van de Winkel JG, Parren PW. Daratumumab, a Novel Therapeutic Human CD38 Monoclonal Antibody, Induces Killing of Multiple Myeloma and Other Hematological Tumors. J Immunol 2011;186:1840-8.

4. Lonial S, Weiss BM, Usmani SZ, Singhal S, Chari A, Bahlis NJ, Belch A, Krishnan A, Vescio RA, Mateos MV, Mazumder A, Orlowski RZ, Sutherland HJ, Blade J, Scott EC, Oriol A, Bardeja J, Gharibo M, Stevens DA, LeBlanc R, Sebag M, Callander N, Jakubowiak A, White D, de la Rubia J, Richardson PG, Lisby S, Feng H, Uhlar CM, Khan I, Ahmadi T, Voorhees PM. Daratumumab monotherapy in patients with treatment-refractory multiple myeloma (SIRIUS): an open-label, randomised, phase 2 trial. Lancet 2016;387:1551-60.

5. https://www.accessdata.fda.gov/drugsatfda_docs/label/2015/761 036Orig1s000lbledt.pdf Accessed Feb 14, 2018.

6. Radocha J, Pour L, Spicka I, Scudla V, Gregora E, Frankova H, Hajek R, Schutzova M, Sykora P, Kessler P, Adamova D, Wrobel M, Sedlarikova L, Sevcikova S, Horinek D, Pelcova J, Brozova L, Jarkovsky J, Maisnar V. Registry of Monoclonal Gammopathies (RMG) in the Czech Republic. Blood 2015;126:4514.

7. Richardson PG, Siegel DS, Vij R, Hofmeister CC, Baz R, Jagannath S, Chen C, Lonial S, Jakubowiak A, Bahlis N, Song K, Belch A, Raje N, Shustik C, Lentzsch S, Lacy M, Mikhael J, Matous J, Vesole D, Chen M, Zaki MH, Jacques C, Yu Z, Anderson KC. Pomalidomide alone or in combination with low-dose dexamethasone in relapsed and refractory multiple myeloma: a randomized phase 2 study. Blood 2014;123:1826-32.

8. Hajek R, Masszi T, Petrucci MT, Palumbo A, Rosinol L, Nagler A, Yong KL, Oriol A, Minarik J, Pour L, Dimopoulos MA, Maisnar V, Rossi D, Kasparu H, van Droogenbroeck J, Yehude DB, Hardan I, Jenner M, Calbecka M, David M, de la Rubia J, Drach J, Gasztonyi Z, Gornik S, Leleu X, Munder M, Offidani M, Zojer N, Rajangam K, Chang YL, San Miguel JF, Ludwig $\mathrm{H}$. A randomized phase III study of carfilzomib vs low-dose corticosteroids with optional cyclophosphamide in relapsed and refractory multiple myeloma (FOCUS). Leukemia 2017;31:107-14.

9. Usmani SZ, Diels J, Ito T, Mehra M, Khan I, Lam A. Daratumumab monotherapy compared with historical control data in heavily pretreated and highly refractory patients with multiple myeloma: An adjusted treatment comparison. Am J Hematol 2017;92:E146-E152.

10. Jelinek T, Maisnar V, Pour L, Spicka I, Minarik J, Gregora E, Kessler P, Sykora M, Frankova H, Adamova D, Wrobel M, Mikula P, Jarkovsky J, Diels J, Gatopoulou X, Vesela S, Besson H, Brozova L, Ito T, Hajek R. Adjusted comparison of daratumumab monotherapy versus realworld historical control data from the Czech Republic in heavily pretreated and highly refractory multiple myeloma patients. Curr Med Res Opin 2018;34(5):775-83. doi: 10.1080/03007995.2017.1410121

11. Blair HA. Daratumumab: A Review in Relapsed and/or Refractory Multiple Myeloma. Drugs 2017;18:2013-24.

12. Lentzsch S, Weisel KC, Mateos M-V, Hungria V, Munder M, Nooka A, Mark T, Quach H, Scott E, Lee J, Sonneveld P, Casneuf T, Chiu C, Qin X, Amin H, Thiyagarajah P, Schecter J, Qi M, Spencer A. Daratumumab, bortezomib and dexamethasone (DVd) vs bortezomib and dexamethasone $(\mathrm{Vd})$ in relapsed or refractory multiple myeloma (RRMM): Efficacy and safety update (CASTOR). J Clin Oncol 2017;35(S15):8036. doi: 10.1200/JCO.2017.35.15_suppl.8036

13. Dimopoulos MA, White DJ, Benboubker L, Cook G, Leiba M, Morton J, Ho PJ, Kim K, Takezako N, Trivedi S, Wu K, Casneuf T, Chiu C, Schecter J, Moreau P. Daratumumab, Lenalidomide, and Dexamethasone (DRd) Versus Lenalidomide and Dexamethasone (Rd) in Relapsed or Refractory Multiple Myeloma (RRMM): Updated Efficacy and Safety Analysis of Pollux. Blood 2017;130(S1):739.

14. Palumbo A, Chanan-Khan A, Weisel K, Nooka AK, Masszi T, Beksac M, Spicka I, Hungria V, Munder M, Mateos MV, Mark TM, Qi M, Schecter J, Amin H, Qin X, Deraedt W, Ahmadi T, Spencer A, Sonneveld P. Daratumumab, Bortezomib, and Dexamethasone for Multiple Myeloma. N Engl J Med,2016;375:754-66.

15. Dimopoulos MA, Oriol A, Nahi H, San-Miguel J, Bahlis NJ, Usmani SZ, Rabin N, Orlowski RZ, Komarnicki M, Suzuki K, Plesner T, Yoon S-S, Ben Yehuda D, Richardson PG, Goldschmidt H, Reece D, Lisby S, Khokhar NZ, O'Rourke L, Chiu C, Qin X, Guckert M, Ahmadi T, Moreau P. Daratumumab, Lenalidomide, and Dexamethasone for Multiple Myeloma. N Engl J Med 2016;375:1319-31.

16. Mateos MV, Dimopoulos MA, Cavo M, Suzuki K, Jakubowiak A, Knop S, Doyen C, Lucio P, Nagy Z, Kaplan P, Pour L, Cook M, Grosicki S, Crepaldi A, Liberati AM, Campbell P, Shelekhova A, Yoon S-S, losava G, Fujisaki T, Garg M, Chiu C, Wang J, Carson R, Crist W, Deraedt W, Nguyen H, Qi M, San Miguel J. Daratumumab plus Bortezomib, Melphalan, and Prednisone for Untreated Myeloma. N Engl J Med 2018;378:518-28.

17. Hofmeister C, Chari A, Cohen YC, Spencer A, Voorhees PM, estell J, Venner CP, Sandhu I, Jenner MW, Williams C, Cavo M, van de Donk NWCJ, Beksac M, Kuppens S, Bandekar R, Neff T, Heuck C, Qi M, Goldschmidt H, Landgren O. Daratumumab monotherapy for patients with intermediate or high-risk smoldering multiple myeloma (SMM): CENTAURUS, a randomized, open-label, multicenter phase 2 study. Blood 2017;35(S1):510.

18. Minarik J, Novak M, Flodr P, Balcarkova J, Mlynarcikova M, Krhovska P, Pika T, Pikalova Z, Bacovsky J, Scudla V. CD38 negative relapse in multiple myeloma after daratumumab based chemotherapy. Eur J Haematol 2017;99:186-9. 